\title{
Perceived Versus Actual Waiting Time: A Case Study Among Cyclists in Enschede, the Netherlands
}

\author{
Tiago Fioreze, Benjamin Groenewolt ${ }^{\dagger}$, Johan Koolwaaij ${ }^{\ddagger}$, Karst Geurs ${ }^{* \star}$ \\ Keywords: smartphone application, signalized intersection, behavioral research, gender, age, actual waiting time, perceived waiting time \\ https://doi.org/10.32866/9636
}

\section{Transport Findings}

This article compares the perceived waiting time of cyclists at signalized
intersections with their actual waiting time. We also examine the relationship
between demographics (age and gender) and perceived waiting time. Data was
collected from May 13 to May 19, $2019(\mathrm{~N}=238)$. Our analysis shows that
cyclists considerably overestimate waiting time by a factor of five. Equally
relevant, we observe that neither age nor gender influences perceived waiting time
since no significant differences were found.

\section{RESEARCH QUESTIONS AND HYPOTHESES}

Traffic lights increase road safety but are also a source of delay for cyclists. Cyclists have a certain aversion when approaching (signalized) intersections because they will likely have to slow down, stop, and subsequently pedal harder to regain their former speed (Fajans and Curry 2001). As a result, cyclists may ignore red lights, which is very risky (Pai and Jou 2014; Fraboni et al. 2016) or avoid traffic lights altogether (Sener, Eluru, and Bhat 2009; Bernardi, La PaixPuello, and Geurs 2018).

In order to reduce waiting times for cyclists, several measures for traffic control have already been introduced or examined in Dutch and other European cities, such as green waves (De Angelis et al. 2019). Recently, the municipality of Enschede, a city with about 160,000 inhabitants in the eastern part of the Netherlands, has introduced SMART Green (Rottier 2017), which gives a faster green light for cyclists who use a dedicated smartphone app called SMART (Self Motivated and Rewarded Travelling). Its introduction is part of a cycling policy plan (Enschede Cycling City 2020) implemented by the municipality of Enschede to increase the modal share of cyclists on short distances $(<7.5 \mathrm{~km})$ from $34 \%$ in 2012 to $40 \%$ in 2020 . The SMART app interacts with several traffic lights throughout Enschede and, by sharing their

\footnotetext{
* University of Twente; Centre for Transport Studies ORCID iD: 0000-0001-7919-6285

† Keypoint Consultancy

$\ddagger$ Mobidot

** University of Twente; Centre for Transport Studies ORCID iD: 0000-0003-0918-8903 Link: https://people.utwente.nl/ k.t.geurs
} 
location, cyclists using the app - referred to now as SMART cyclists - have a greater chance of shortening their waiting time when approaching certain traffic lights.

In this article, we examine the differences between actual and perceived waiting times of SMART cyclists at signalized intersections in Enschede and the variation between different socio-economic groups. While there are published accounts of perceived waiting times among drivers (Vreeswijk, Van Berkum, and Van Arem 2015; Mao et al. 2018), it is our belief that this is the first work that shows how cyclists perceive waiting times at signalized intersections. Our hypothesis is that cyclists' perceived waiting time is higher than their actual waiting time. Furthermore, some studies in the literature show that gender and age may play a role with respect to perceived waiting times (Fan, Guthrie, and Levinson 2016; Cascajo et al. 2018). Our hypothesis is that males and younger people have higher perceived waiting times than females and elderly people.

\section{METHODS AND DATA}

Two sources of data are used for this experiment. First, a short in-app survey was conducted among SMART cyclists for obtaining their perceived waiting time and demographics (age and gender). Only SMART cyclists who regularly use the SMART app and cycle through the streets of Enschede were invited to fill out the survey, resulting in 238 survey respondents out of 1,949 invited SMART app users.

Second, the actual waiting time per traffic light was automatically measured by means of the SMART app and stored on the MoveSmarter platform (Thomas et al. 2018). The stored data is based on one week of continuous measuring of SMART cyclists' whereabouts. Moreover, the data contain the number of traffic light passages along with the percentage of times cyclists had to wait, and the average waiting time in seconds. Considering the 1,949 SMART app users invited for our experiment, approximately 31,000 traffic light passages were observed during the one-week period. Furthermore, $42 \%$ of those passages resulted in cyclists having to wait for the green light with an average waiting time of 26 seconds.

In addition, all bicycle trips collected by the SMART app were recorded as map matching-based entries and circles with a radius of 50 meters were drawn around traffic lights. When a trip entry intersected with a circle, a routine was used to calculate whether a cyclist had lost time at that traffic light. Lost time is calculated as the time the cyclist needs to pass through the circle minus the cyclist's distance in the circle divided by their personal average cycling speed.

Figure 1 shows an example of a cyclist with an average speed of five meters per second taking 80 seconds to cross the circle. Because it is expected to take 20 seconds to do so, it is assumed that the cyclist had to wait 60 seconds for the green light. 


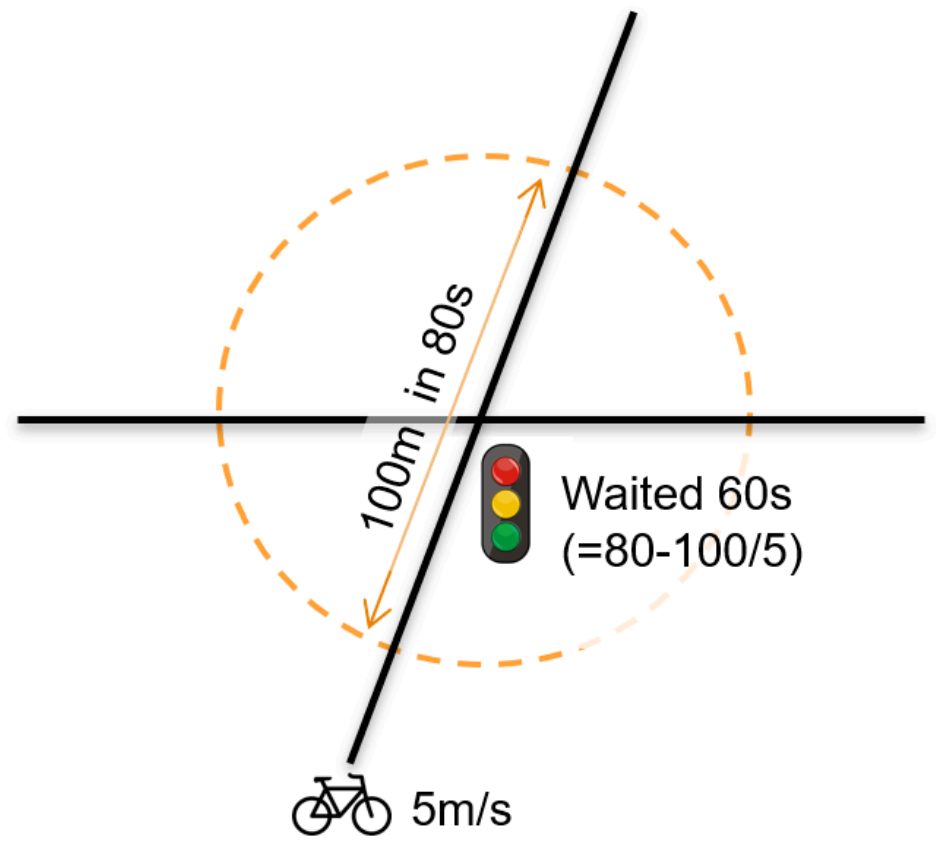

Figure 1: Depiction of How Lost Time is Calculated in Our Analysis

Table 1: Descriptive Statistics for Perceived and Actual Waiting Time Variables

\begin{tabular}{llll}
\hline Variables & N & Mean & Std. Deviation \\
\hline Perceived Waiting Time & 238 & 76 & 60 \\
Actual Waiting Time & 202 & 15 & 7
\end{tabular}

SMART complies with EU General Data Protection Regulation; the data is securely stored and all analysis is done on aggregated datasets where the data cannot be traced back to individual users. This method ensures that participants remain completely anonymous. In this research, we only use the lost time statistics of each traffic signal aggregated over the one-week period. Out of 238 survey respondents, we observed the actual waiting times of 202 of them. The remaining 36 apparently did not use the app during that period. As a result, only the actual waiting times of 202 SMART cyclists are considered in our analysis.

\section{FINDINGS}

The mean and standard deviation of the perceived and actual waiting times (both measured in seconds) are estimated and shown in Table 1.

Given that both variables are not normally distributed, the Wilcoxon signedrank test was used to examine if perceived and actual waiting times significantly differ $(\alpha=.05)$. The SMART cyclists' perceived waiting time was approximately five times higher than their actual waiting time; $\mathrm{Z}=-11.92, \mathrm{p}$ $<.001$. 


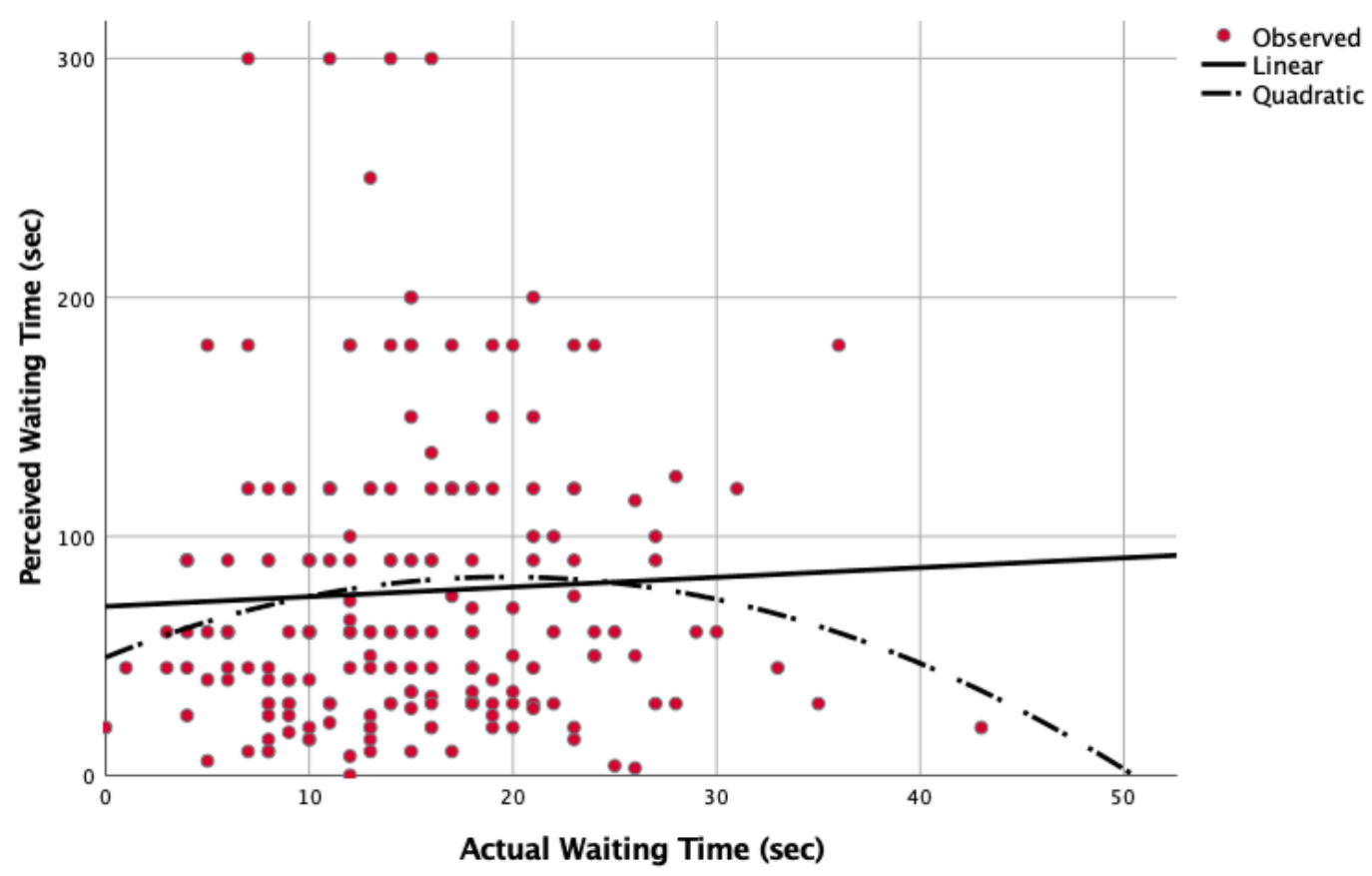

Figure 2: Scatter Plot of Perceived Waiting Time and Actual Waiting Time

When looking at the mean perceived waiting time, it appears that most cyclists considerably overestimate their waiting time, which confirms our hypothesis. This shows traffic lights are perceived by cyclists as an important source of delay. However, we observed that most cyclists estimated waiting time in round numbers (e.g., 30 seconds, 45 seconds, 60 seconds and so forth), given that the frequencies of those numbers were higher than the rest. It comes therefore as no surprise that, based on its large standard deviation, the perceived waiting time varied largely among cyclists and it may consequently be rather biased.

Regression analyses were also performed to test if we could predict perceived waiting time from actual waiting time. Linear regression and a nonlinear (quadratic function) regression have been tested but none were successful (note that a regression analysis with a logarithmic transformation to the dependent variable was also tested without success). Figure 2 and Table 2 summarize the results, which are in line with the literature. That is, subjective time does not necessarily bear a straightforward connection to physical objective time (Tenenboim and Shiftan 2016). Similarly, waiting time in (public) transport is also typically overestimated (Van Hagen, Galetzka, and Pruyn 2014).

Next, a Pearson product-moment correlation coefficient was computed to assess the correlation between the cyclists' age and their perceived waiting time. Our analysis shows that there is no correlation between the two variables, $\mathrm{r}=$ $.068, \mathrm{n}=185, \mathrm{p}=.356$. A scatter plot summarizes the results (Figure 3 ). 
Table 2: Model Summary and Parameter Estimates for the Linear and Nonlinear (Quadratic Function) Regressions ${ }^{\mathrm{a}}$

\begin{tabular}{lllllllll}
\hline \multirow{2}{*}{ Dependent Variable } & \multicolumn{3}{c}{ Perceived Waiting Time } & \multicolumn{3}{c}{ Podel Summary } & \multicolumn{3}{c}{ Parameter Estimates } \\
\hline Equation & R Square & F & df1 & df2 & Sig. & Constant & b1 & b2 \\
\hline Linear & 0,002 & 0,460 & 1 & 200 & 0,499 & 70,686 & 0,406 & \\
Quadratic & 0,015 & 1,506 & 2 & 199 & 0,224 & 49,346 & 3,426 & $-0,087$
\end{tabular}

${ }^{a}$ The independent variable is actual waiting time.

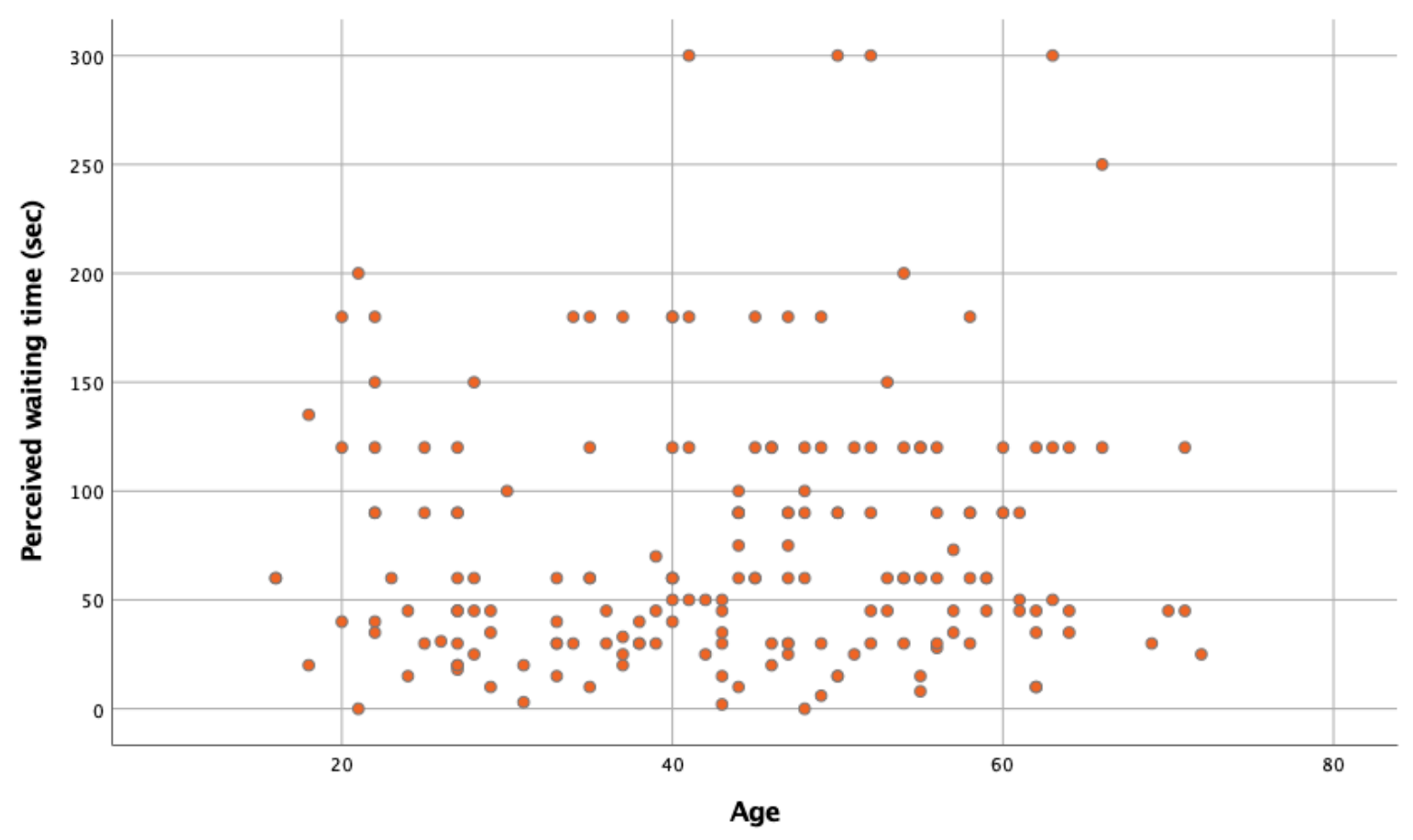

Figure 3: Scatter Plot of Perceived Waiting Time by Age

Finally, an Independent Samples $t$-Test was conducted to compare perceived waiting time between male and female cyclists. There was no significant difference in the perceived waiting time for male cyclists $(M=71, S D=47)$ and female cyclists $(M=86, S D=71) ; t(92.289)=-1.37, p=.175$. Similar to results based on age, these results suggest that gender does not have an effect on perceived waiting time. Specifically, our results suggest that female and male cyclists perceive waiting times the same way.

\section{ACKNOWLEDGMENTS}

The authors would like to thank the municipality of Enschede and Mobidot for making this experiment possible. 


\section{REFERENCES}

Bernardi, Silvia, Lissy La Paix-Puello, and Karst Geurs. 2018. "Modelling Route Choice of Dutch Cyclists Using Smartphone Data." Journal of Transport and Land Use 11 (1): 883-900. https://doi.org/10.5198/jtlu.2018.1143.

Cascajo, Rocio, Elena Lopez Suárez, Fernando Herrero, and Andres Monzon. 2018. "User Perception of Transfers in Multimodal Urban Trips: A Qualitative Study.” International Journal of Sustainable Transportation 13 (6): 393-406. https://doi.org/10.1080/15568318.2018.1476632.

De Angelis, Marco, Arjan Stuiver, Federico Fraboni, Gabriele Prati, Víctor Marín Puchades, Filippo Fassina, Dick de Waard, and Luca Pietrantoni. 2019. "Green Wave for Cyclists: Users' Perception and Preferences.” Applied Ergonomics 76 (April): 113-21. https://doi.org/10.1016/ j.apergo.2018.12.008.

Fajans, J., and M. Curry. 2001. "Why Bicyclists Hate Stop Signs." ACCESS Magazine 1 (18): 28-31. https://www.accessmagazine.org/spring-2001/bicyclists-hate-stop-signs/.

Fan, Yingling, Andrew Guthrie, and David Levinson. 2016. "Waiting Time Perceptions at Transit Stops and Stations: Effects of Basic Amenities, Gender, and Security." Transportation Research Part D: Policy and Practice 88 (June): 251-64. https://doi.org/10.1016/j.tra.2016.04.012.

Fraboni, Federico, Victor Marín Puchades, Marco De Angelis, Luca Pietrantoni, and Gabriele Prati. 2018. "Red-Light Running Behavior of Cyclists in Italy: An Observational Study." Accident Analysis $\sigma^{2}$ Prevention 120 (November): 219-32. https://doi.org/10.1016/j.aap.2018.08.013.

Fraboni, Federico, Víctor Marín Puchades, Marco De Angelis, Gabriele Prati, and Luca Pietrantoni. 2016. "Social Influence and Different Types of Red-Light Behaviors among Cyclists." Frontiers in Psychology 7 (November). https://doi.org/10.3389/fpsyg.2016.01834.

Mao, Yan, Wuhong Wang, Chenxi Ding, Weiwei Guo, Xiaobei Jiang, Martin Baumann, and Geert Wets. 2018. "A Measurement to Driving Situation Awareness in Signalized Intersections." Transportation Research Part D: Transport and Environment 62 (July): 739-47. https://doi.org/ 10.1016/j.trd.2018.05.001.

Pai, Chih-Wei, and Rong-Chang Jou. 2014. "Cyclists' Red-Light Running Behaviours: An Examination of Risk-Taking, Opportunistic, and Law-Obeying Behaviours." Accident Analysis E Prevention 62 (January): 191-98. https://doi.org/10.1016/j.aap.2013.09.008.

Rottier, J.P. 2017. “Ook in Enschede Krijgen Fietsers Met App Sneller En Langer Groen, Verkeersnet." Het Vakmagazine Voor de Mobiliteitsprofessional. https://www.verkeersnet.nl/fiets/ 24955/ook-enschede-krijgen-fietsers-app-sneller-en-langer-groen/.

Sener, Ipek N., Naveen Eluru, and Chandra R. Bhat. 2009. "An Analysis of Bicycle Route Choice Preferences in Texas, US.” Transportation 36 (5): 511-39. https://doi.org/10.1007/ s11116-009-9201-4.

Tenenboim, Einat, and Yoram Shiftan. 2016. "Accuracy and Bias of Subjective Travel Time Estimates.” Transportation 45 (3): 945-69. https://doi.org/10.1007/s11116-016-9757-8.

Thomas, Tom, Karst T. Geurs, Johan Koolwaaij, and Marcel Bijlsma. 2018. "Automatic Trip Detection with the Dutch Mobile Mobility Panel: Towards Reliable Multiple-Week Trip Registration for Large Samples.” Journal of Urban Technology 25 (2): 143-61. https://doi.org/ $\underline{10.1080 / 10630732.2018 .1471874 .}$.

Van Hagen, M., M. Galetzka, and A.T. Pruyn. 2014. "Waiting Experience in Railway Environments." Journal of Motivation, Emotion, and Personality 2 (2): 41-55. https://doi.org/ $\underline{10.12689 / \text { jmep.2014.305. }}$. 
Vreeswijk, Jaap, Eric Van Berkum, and Bart Van Arem. 2015. "Evaluating Awareness and Perception of Waiting Time at Signalized Intersections: Field Study.” Transportation Research Record: Journal of the Transportation Research Board 2518 (1): 86-94. https://doi.org/10.3141/ 2518-12. 